\title{
Variational Monte Carlo studies of gossamer superconductivity
}

\author{
Siegfried Guertler, ${ }^{1}$ Qiang-Hua Wang, ${ }^{2}$ and Fu-Chun Zhang ${ }^{1}$ \\ ${ }^{1}$ Department of Physics and Center of Theoretical and Computational Physics, The University of Hong Kong, Hong Kong, China \\ ${ }^{2}$ Department of Physics and National Laboratory of Solid State Microstructures, Nanjing University, Nanjing 210093, China
}

(Received 23 September 2008; revised manuscript received 9 March 2009; published 27 April 2009)

\begin{abstract}
We use a partially Gutzwiller projected BCS $d$-wave wave function with an antiferromagnetic-weighting factor to study the ground-state phase diagram of a half-filled Hubbard-Heisenberg model in a square lattice with nearest-neighbor hopping $t$ and a diagonal hopping $t^{\prime}$. The calculations are carried out by using variational Monte Carlo method which treats the Gutzwiller projection explicitly. At large on-site Coulomb interaction $U$, the ground state is antiferromagnetic. As $U$ decreases, the ground state becomes superconducting and eventually metallic. The phase diagram is obtained by extensive calculations. As compared to the strong effect of $U / t$, the phase boundaries turn out to be less sensitive to $t^{\prime} / t$. The result is consistent with the phase diagram in layered organic conductors and is compared to the earlier mean-field result based on the Gutzwiller approximation.
\end{abstract}

DOI: 10.1103/PhysRevB.79.144526

PACS number(s): 74.70.Kn, 71.30.+h, 02.70.Ss, 74.20.Mn

\section{INTRODUCTION}

High-temperature superconductivity remains to be an exciting and rich field. One of the interesting proposals is Anderson's resonating valence bond (RVB) state. ${ }^{1-3}$ In the RVB theory, the parent compound is an insulator at half electron filling or one electron per $\mathrm{Cu}$ site, and chemical doping is essential to introduce charge carriers to lead to superconductivity. The mathematics of the RVB theories therefore is in a Hilbert space which completely projects out the on-site double-occupied electron states. At the half filled, there is exactly one electron per lattice site and the charge degree of freedom is totally frozen, resulting in a Mott insulator.

Another interesting class of materials in the context of strongly correlated systems is the layered organic conductors, ${ }^{4-7}$ which may undergo a phase transition from an insulator to a superconducting (SC) state by applying pressure. $^{4}$ Since these materials are effectively at half filling, ${ }^{8}$ the phase transition is due to the competition between the Coulomb interaction and kinetic bandwidth, the latter of which is tuned by pressure instead of chemical doping. There have been several related theoretical works on layered organic superconductors in recent years. ${ }^{8-19}$ The mathematics of the SC state may be described by a partially Gutzwiller projected BCS state, ${ }^{11,20}$ instead of the complete projection as in the RVB theory. We shall refer to this partially Gutzwiller projected BCS state as a Gossamer superconductor, a phrase first introduced by Laughlin ${ }^{21,22}$ originally in the context of high-temperature superconductors. Gossamer superconductivity refers to those SC states with a dilute superfluid density. The partial Gutzwiller projection allows charge fluctuations even at half filling. Zhang ${ }^{20}$ proposed that in this case an effective model is the HubbardHeisenberg model which includes the standard kinetic energy, the on-site Coulomb repulsion, as well as the antiferromagnetic (AFM) spin exchange. The idea was applied to the study of $\kappa$-(BEDT-TTF $)_{2} X$ by Gan et al., ${ }^{11}$ where the Gutzwiller approximation was used to replace the partial Gutzwiller projection by a set of renormalized factors and the resulted renormalized Hamiltonian was then studied by a mean-field theory. The finding is a phase diagram distinguishing three phases: normal metal, superconductor, and antiferromagnet.

In this paper, we shall study the phase diagram of an effective Hubbard-Heisenberg model related to $\kappa$-(BEDT-TTF $)_{2} X$ by using variational Monte Carlo (VMC) method on a trial-wave function. Note that we introduce a Heisenberg spin-coupling term to take into account the antiferromagnetic interaction explicitly because the variational state we use cannot properly treat antiferromagnetic interaction in a pure Hubbard model. The order parameters for the $d$-wave superconductivity and for the antiferromagnetism are calculated directly. We obtain a phase diagram consistent with the experiments, providing further support to the scenario of the Gossamer superconductivity to describe the layered organic conductors. Interestingly, our numerical calculation of the SC order parameter suggests a relatively highsuperfluid density near the phase boundary to the AFM insulator. The results from our VMC calculations also provide support to the earlier mean-field results based on the Gutzwiller approximation, ${ }^{11}$ although we find a less sensitive role of $t^{\prime}$.

\section{MODEL, TRIAL-WAVE-FUNCTION, AND METHOD}

We study a Hubbard-Heisenberg model in a twodimensional lattice illustrated in Fig. 1. The Hamiltonian is given by

$$
H=\sum_{i} U n_{i \uparrow} n_{i \downarrow}-\sum_{\langle i, j\rangle \sigma} t_{i j} c_{i \sigma}^{\dagger} c_{j \sigma}+\text { H.c. }+\sum_{(i, j)} J S_{i} \cdot S_{j} .
$$

Here $c_{j \sigma}$ is the electron annihilation operator of an electron with spin $\sigma$ on-site $i, \vec{S}_{i}$ is the spin-1/2 operator at site $i$, and $n_{i \sigma}=c_{i \sigma}^{\dagger} c_{i \sigma}$. The sum $(i, j)$ is over the nearest-neighbors (n.n.) pairs on the square lattice and the sum over $\langle i, j\rangle$ is over both the n.n. pairs and the diagonal bonds (dashed lines in Fig. 1). We set the n.n. hopping $t_{i, j}=t=1$ as the energy unit, fix the spin exchange $J$ as $J / t=0.5$, and treat the diagonal-hopping integral $t_{i, j}=t^{\prime}$ and the on-site repulsion $U$ as tuning param- 


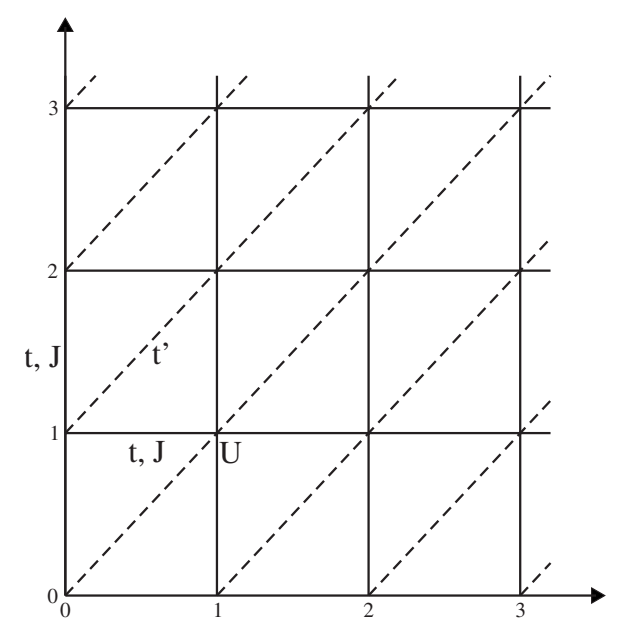

FIG. 1. Illustration of the lattice for Hamiltonian (1) studied in this paper. $t$ and $J$ are hopping and spin-exchange coupling between nearest-neighbor pairs (solid lines) and $t^{\prime}$ is the hopping integral along a diagonal direction (dashed lines).

eters. In our numerical calculations, we consider a $L$ by $L$ lattice and use a periodic boundary condition along the $x$ direction and antiperiodic boundary condition along the $y$ direction.

Our trial-wave-function reads

$$
|\Psi\rangle=\exp \left(\beta \sum_{\langle i, j\rangle} S_{i}^{z} S_{j}^{z}\right) \prod_{i}\left(1-\alpha n_{i \uparrow} n_{i \downarrow}\right)\left|\Psi_{N_{e}}\right\rangle,
$$

where $\left|\Psi_{N}\right\rangle$ is the BCS-wave function projected to the subspace with the fixed number of particles $N_{e}$ as defined in Eq. (3) below. In Eq. (2), we introduce two variational parameters $\alpha$ and $\beta$ to control the partial Gutzwiller projection and the AFM correlation, respectively. The BCS-wave function in the fixed particle formalism has the following form in real space:

$$
\left|\Psi_{N_{e}}\right\rangle=\left[\sum_{j_{\downarrow}, l_{\uparrow}} a\left(R_{j \downarrow}-R_{l \uparrow}\right) c_{l, \uparrow}^{\dagger} c_{j, \downarrow}^{\dagger}\right]^{N_{e} / 2}|0\rangle,
$$

where $R_{j \sigma}$ is the spatial position of an electron with spin $\sigma$ at the lattice site $j$, and the sum is over all the pairs of a spin-up electron at site $j$ and a spin-down electron at site $l$. Here $a(\boldsymbol{r})$ is the amplitude of the wave function, which is the Fourier transform of $a(\boldsymbol{k})=v_{k} / u_{k}$, with $u_{k}$ and $v_{k}$ given in the usual BCS-wave function,

$$
\left|\Psi_{\mathrm{BCS}}\right\rangle=\prod_{\boldsymbol{k}}\left(u_{\boldsymbol{k}}+v_{\boldsymbol{k}} c_{\boldsymbol{k} \uparrow}^{\dagger} c_{-\boldsymbol{k} \downarrow}^{\dagger}\right)|0\rangle .
$$

We have $e^{1,23}$

$$
\begin{gathered}
a(\boldsymbol{r})=\sum_{k} a_{k} \cos (\boldsymbol{k r}), \\
a_{k}:=\frac{\Delta(\boldsymbol{k})}{\xi_{k}+\sqrt{\xi_{k}^{2}+\Delta(\boldsymbol{k})^{2}}} .
\end{gathered}
$$

Following the previous literature on the pairing symmetry for the model, ${ }^{10,11}$ we focus here on the $d_{x^{2}-y^{2} \text {-wave pairing }}$ state, where $\Delta(\boldsymbol{k})$ and $\xi_{\boldsymbol{k}}$ have the following forms:

$$
\begin{gathered}
\Delta(\boldsymbol{k})=\Delta\left[\cos \left(k_{x}\right)-\cos \left(k_{y}\right)\right], \\
\xi_{\boldsymbol{k}}=-2 t\left[\cos \left(k_{x}\right)+\cos \left(k_{y}\right)\right]-2 t_{v}^{\prime} \cos \left(k_{x}+k_{y}\right)-\mu,
\end{gathered}
$$

where $\Delta, \mu$, and $t_{v}^{\prime}$ are variational parameters in the theory. Note that $t_{v}^{\prime} \neq t^{\prime}$ in general due to the spin coupling term in the Hamiltonian. The advantage of the above trial-wave function is that the SC and AFM order can be treated on an equal footing. It turns out that a small value of $\beta$ improves the energy of the SC state, while a sufficiently large value of $\beta$ leads to AFM long-range ordering. We measure the staggered magnetization to quantitatively study the AFM phase,

$$
m=\sqrt{\frac{1}{N(N-1)} \sum_{\langle i, \vec{r}\rangle}\left\langle S_{i}^{z} S_{i+\vec{r}}^{z}\right\rangle(-1)^{r_{x}+r_{y}},}
$$

where $N$ is the number of the lattice sites, and the sum is over all the $N(N-1)$ pairs between sites $i$ and $i+\vec{r}$ on the lattice. To measure the SC long-range order, we introduce a pair-correlation function,

$$
\begin{gathered}
\phi_{i, j}=F_{i} F_{j}^{\dagger}, \\
F_{i}=\frac{1}{4} \sum_{\tau} b_{i, i+\tau}(-1)^{\tau_{y}}, \\
b_{i, i+\tau}=\frac{1}{\sqrt{2}}\left(c_{i \downarrow} c_{i+\tau \uparrow}-c_{i \uparrow} c_{i+\tau \downarrow}\right),
\end{gathered}
$$

where $b_{i, i+\tau}$ is a spin-singlet bond between the two sites $i$ and $i+\tau$, and $\tau= \pm \hat{x}, \pm \hat{y}, \tau_{y}=0$ for $\tau= \pm \hat{x}$, and $\tau_{y}= \pm 1$ for $\tau= \pm \hat{y} . F_{i}$ describes a $d$-wave singlet bond around site $i$. The off-diagonal long-range order parameter for the $d$-wave pairing can be measured by the quantity at $R \rightarrow \infty,{ }^{26}$

$$
\phi(\vec{R})=\frac{1}{N} \sum_{i}\left\langle\phi_{i, i+\vec{R}}\right\rangle,
$$

where the sum is over all the lattice sites. In our calculations on the finite-size systems, we choose $\vec{R}=(L / 2, L / 2)$, the largest displacement on the lattice of $L$ by $L$ with $L$ up to 10 . As a third quantity, we measure the average double occupation $d$, which is the average amount of double-occupied sites over the lattice sites.

To simplify the variational procedure, we will fix $\mu$ in the calculations with the reasons given below. It has been $\operatorname{argued}^{23}$ that $\Delta$ and $\mu$ are not independent on the variational calculations for the $t-J$ model. We found that the results are essentially insensitive to $\mu$ for the present model. In contrast, $t_{v}^{\prime}$ is an important variational parameter here. The groundstate energies and the ground-state phase are sensitive to $t_{v}^{\prime}$ over a wide parameter range. By fixing $\mu$, we have then four variational parameters $\left(\Delta, t_{v}^{\prime}, \alpha, \beta\right)$ in our calculations to determine the phase diagram in the parameter space of $U$ and $t^{\prime}$.

There are two sources of error bars in our numerical calculations within the variational approach: one is from the statistical errors and the other is due to the discreteness of the variational parameters in our calculations. In our simulation, we start with several different initial configurations and then 


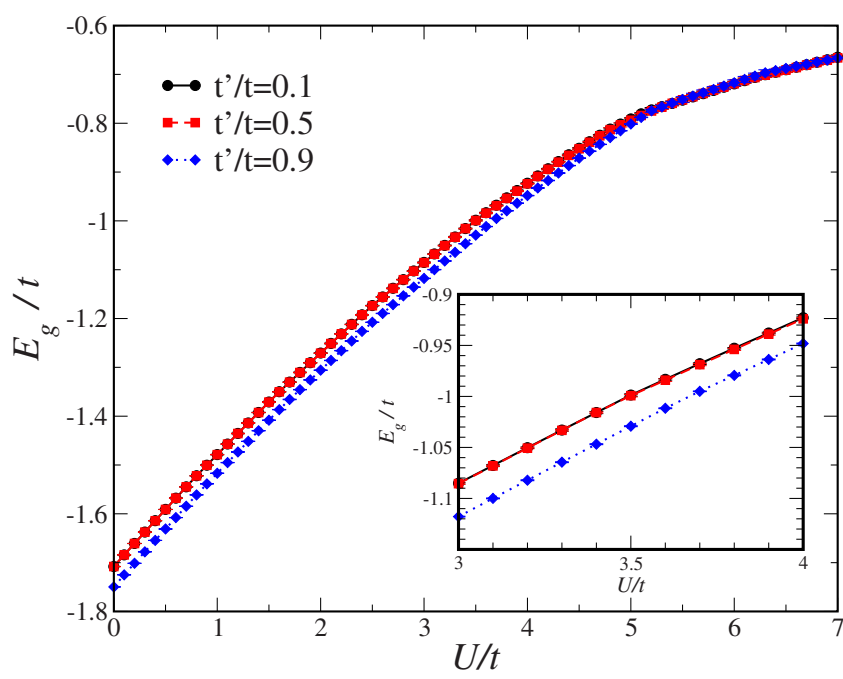

FIG. 2. (Color online) Variational ground-state energy $E_{g}$ of Hamiltonian (1) as a function of $U / t$ for $J / t=0.5$ and various values of $t^{\prime} / t$. The inset is an enlarged figure for the energy.

average our numerical measurements over those simulations. The error bars obtained in these averages are found to be 1 order of magnitude smaller than the error bars described below. We consider the possible values for the variational parameters and divide them into small slices. Then we perform VMC for all combinations of this "mesh." After obtaining an optimal set of variational parameters in this mesh for a particular set of $U / t$ and $t^{\prime} / t$, we develop a local mesh for nearby values of the tuning parameters. From the spacing of our mesh, we obtain the error bars for the variational parameters. The results and the error bars we present in this paper are essentially due to the finite elements we choose in the variational parameters.

\section{RESULTS AND DISCUSSION}

In this section, we present our results on the variational ground-state, the corresponding variational parameters, the SC, and AFM long-range orders of Hamiltonian (1) in the parameter space of $U / t$ and $t^{\prime} / t$. Since the phase of the ground state is much more sensitive to the on-site repulsion $U$ than to the diagonal hopping $t^{\prime}$, we focus our study on three values of $t^{\prime}$, with $t^{\prime} / t=0.1,0.5$, and 0.9 . With respect to the possible connection to $\kappa$-(BEDT-TTF $)_{2} X$, the value of $t^{\prime} / t$ depends on the compound $X$ and the pressure applied. The expected influence of applying pressure on the values for $t^{\prime} / t$ and $U / t$ is schematically indicated in Fig. 4. For the class of $\kappa$-(BEDT-TTF $)_{2} X$, the $t^{\prime} / t$ ratio is in the range of $t^{\prime} / t=0.4-0.8$. Estimates for some particular cases under ambient pressure are $t^{\prime} / t=0.7$ for $X=\mathrm{Cu}(\mathrm{NCS})_{2}$ and $t^{\prime} / t=0.8$ for $X=\mathrm{Cu}\left[\mathrm{N}(\mathrm{CN})_{2}\right] \mathrm{Br}$. These values are obtained by extended Hückel calculations. ${ }^{24}$ In Fig. 2, we plot the obtained ground-state energies as functions of $U$ for different values of $t^{\prime}$. The corresponding optimized variational parameters and long-range SC and AFM order parameters as functions of $U$ for three sets of values of $t^{\prime}$ are plotted in Fig. 3. The simulations are carried out on lattice sizes of $L=6,8$, and 10, as indicated in the figure. Before we discuss the results, we

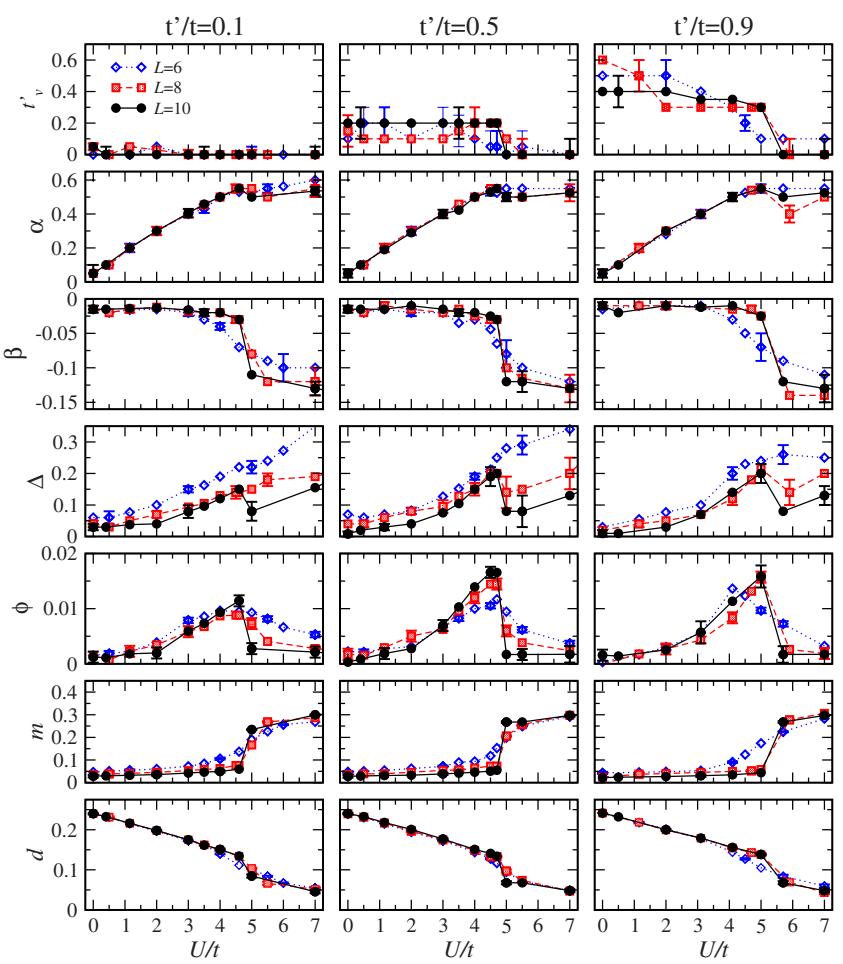

FIG. 3. (Color online) The ground-state variational parameters $t_{v}^{\prime}$ [Eq. (7)], $\alpha$ [partial projection, Eq. (2)], $\beta$ [AFM weighting, Eq. (2)], and $\Delta$ [pairing amplitude, Eq. (6)] as functions of $U / t$ for $t^{\prime} / t=0.1$ (left), $t^{\prime} / t=0.5$ (mid), and $t^{\prime} / t=0.9$ (right). $J / t=0.5$ is fixed. Also plotted are the measured $d$-wave SC order parameter $\phi$ [Eq. (9)], the staggered magnetization $m$ [Eq. (8)], and the average double occupation of sites $d$. The lattice size is $L \times L$, with $L=6,8$, and 10 . The selected error bars shown are typical, due to the finite parameter spacing in our calculations.

note that the spin coupling term in Eq. (1)) is to account the virtual hopping process in the Hubbard model, which is derived at the large $U$ limit. The present study may be of relevance to the Hubbard model only at large $U$ but not at small $U$. Our main interest will be at large or intermediate values of $U$, and the interpretation of our results at small $U$ to the Hubbard model should be cautious.

Before we discuss general features, we briefly discuss the obtained variational parameter $t_{v}^{\prime}$, which is to optimize the kinetic energy due to the presence of the diagonal hopping integral $t^{\prime} . t_{v}^{\prime}$ increases as $t^{\prime}$ increases, but $t_{v}^{\prime}$ is significantly smaller than $t^{\prime}$ as we can see from the first row in Fig. 3. At large $U, t_{v}^{\prime}$ becomes zero or very small. This may be understood as a result of the AFM ground-state with commensurate wave vector $(\pi, \pi)$, since a finite $t_{v}^{\prime}$ does not match the AFM state and is not preferred. ${ }^{25}$

As $U$ increases from zero, the projection parameter $\alpha$ increases from around 0.05 , indicating a graduate increase in Gutzwiller projection, while the weighting factor parameter $\beta$ changes little at small $U$, but changes rapidly around $U$ $\approx 5$. The mean-field pairing amplitude parameter $\Delta$ changes slowly at small $U$, but increases rapidly starting from around $U=2$, then reaches a maximum at around $U=4.5$ and drops at larger $U$. The ground-state properties are best seen in the measurement of the SC order parameter $\phi$ and AFM-order 

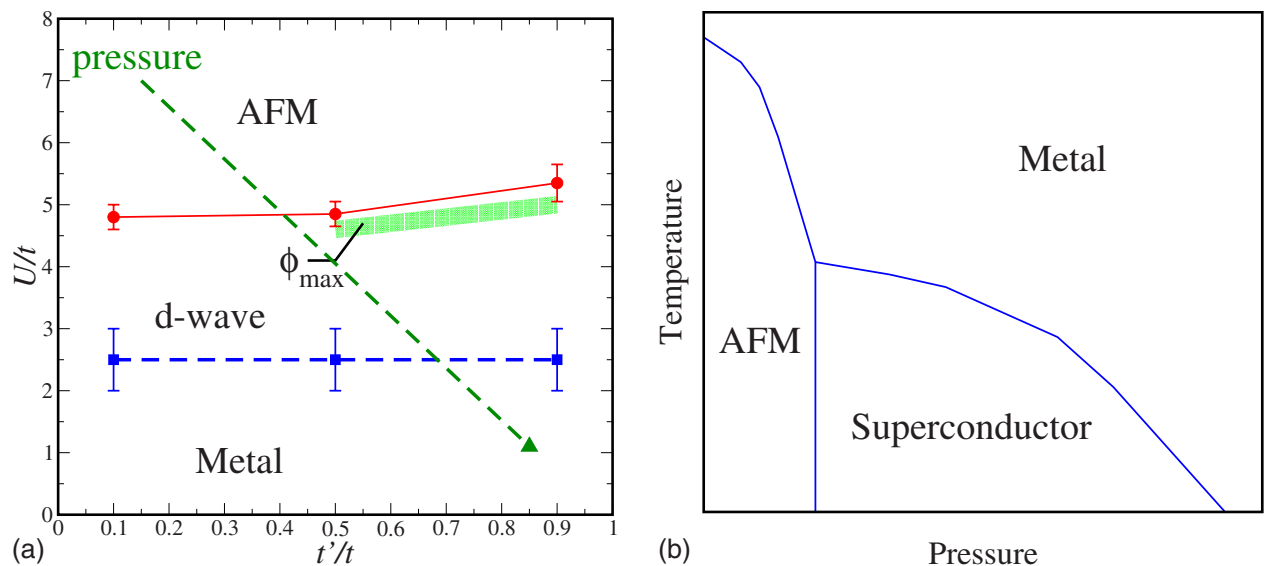

FIG. 4. (Color online) (a) Phase diagram of the ground state of Hamiltonian (1) obtained from our VMC calculations: metal (metallic phase), $d$-wave SC phase, and AFM phase. The region with large SC order parameter is indicated by a thick line and marked with $\phi_{\text {max }}$. The values of the order parameters for the $d$ wave and for the AFM phases can be found in Fig. 3. The arrow indicates the schematic flow of the parameters when pressure is applied. (b) Phase diagram of organic superconductors in parameter space of temperature and pressure.

parameter $m$. Qualitatively there are three regions as $U$ increases. At small $U$, both $\phi$ and $m$ are very small or essentially zero, indicating a metallic state. At intermediate $U, \phi$ increases monotonically with $U$, while $m$ remains small, indicating a SC state. As $U$ further increases, $\phi$ drops sharply, while $m$ increases rapidly. We may identify this phase as the AFM phase without the SC order. The small but nonzero values of $\phi$ and $m$ in the nonordered states may be explained as finite-size effect, although a systematic scaling analysis is difficult due to the small sizes we have studied. The measured double occupation $d$ decreases monotonically, with a sudden drop at the transition between the SC and AFM phases. Note that this quantity continues to drop, even though $\alpha$ saturates after this phase transition. The reason for this is that $\beta$ effects this quantity too, as a perfect AFM state has no double occupation. The measured value of $d$ resembles some consistency with a Mott transition. The above features are qualitatively similar for $t^{\prime} / t=0.1,0.5$, and 0.9 . This is somewhat different from the early analytic calculations by using Gutzwiller approximations on the projectedwave functions, where $t^{\prime}$ is found to suppress the AFM phase. We note that while the onset for the SC phase is similar for different $t^{\prime}$, the magnitude of the SC-order parameter is much bigger for the $t^{\prime} / t=0.5$ and $t^{\prime} / t=0.9$. As we can see from the figure, the largest SC order parameter $\phi$ is found near the boundary to the AFM phase. At $t^{\prime}=0$, we expect model (1) to have instability toward a commensurate AFM state for any finite $U$.

In Fig. 4, we plot the phase diagram of model (1) obtained within our variational wave functions. While the phase boundary between SC and AFM can be found easily by considering one point clearly belonging to the AFM and one point clearly belonging to the SC phase, between SC and metallic phase we have to use an arbitrary value to define the phase boundary, as the onset of the SC-order parameter $\phi$ is not so sharp. We choose $\phi<0.004$ as our criteria classifying the phase to be SC. The error bars in this diagram reflect within which area we have uncertainty that a point would be in either of the two phases considered. Comparing the phase diagram obtained in the VMC method with the previous re- sult by using the renormalized mean-field theory, ${ }^{11}$ they qualitatively agree with each other in the sense that both give the three phases, and overall features are similar. However, there are two differences. First, while both of the methods give the transition point between the SC and AFM phases to increase when $t^{\prime} / t$ increases, in the VMC calculation, the effect is not as big as in the earlier Gutzwillerapproximation-based calculation. In our calculation, if we consider a fixed and nonzero $t_{v}^{\prime}$ instead of a variational one, we would in fact get a slope close to the one reported by Gan et al. The second difference is that our VMC suggests the onset for superconductivity to be at $U=2.5$ for all cases, while Gan et al. found this phase boundary to change considerably when tuning $t^{\prime} / t$. It is difficult to conclude which approximation gives a more reliable result, thus only the qualitative phase diagram common to both methods should be considered reliable. We also note that our model does not represent the Hubbard model correctly for small values of $U$. For comparison with the experiments, we plot a schematicphase diagram for the layered organic conductors at the right panel of Fig. 4.

In summary, we have presented the results of VMC calculations for a recently suggested model for Gossamer superconductivity. Our trial-wave function has the ingredient to describe metallic, AFM, and SC states. This was archived by means of using Jastrow factors for partial Gutzwiller projection and AFM weighting. We showed that the VMC result is consistent with experiments and supports the previously suggested analytical variational calculations qualitatively, as we were able to identify the three expected phases, with the help of measurements of the order parameters for AFM and SC. The exact transition line between SC and metallic phases and between SC and AFM phases differs from the one found previously.

\section{ACKNOWLEDGMENTS}

The VMC calculations have been carried out on the HPCPOWER-cluster, the WinHPC-cluster and the windows- 
condor all powered by HKU's computer center. We wish to thank Masao Ogata for the advise on VMC. Further we wish to thank Kwan Wing Keung for help in parallelization of the code and customization of the code for the windows-condorsystem. This work was partly supported by Hong Kong's
RGC grant. The work in Nanjing was supported by NSFC under Grant No. 10325416, the Ministry of Science and Technology of China (under Grants No. 2006CB921802 and No. 2006CB601002), and the 111 Project (under Grant No. B07026).
${ }^{1}$ P. W. Anderson, Science 235, 1196 (1987).

${ }^{2}$ P. W. Anderson, P. A. Lee, M. Randeria, T. M. Rice, N. Trivedi, and F. C. Zhang, J. Phys.: Condens. Matter 16, R755 (2004).

${ }^{3}$ P. A. Lee, N. Nagaosa, and X. G. Wen, Rev. Mod. Phys. 78, 17 (2006).

${ }^{4}$ D. Jerome, Science 252, 1509 (1991).

${ }^{5}$ R. H. McKenzie, Science 278, 820 (1997).

${ }^{6}$ T. Shiguro, K. Yamaji, and G. Saito, Organic Superconductors, 2nd ed. (Springer, Berlin, 1998).

${ }^{7}$ M. Lang and J. Mueller, in Superconductivity, edited by K. H. Benneman and J. B. Ketterson (Springer, Berlin/Heidelberg, 2008), pp. 1155-1228.

${ }^{8}$ H. Kino and H. Fukuyama, J. Phys. Soc. Jpn. 64, 2726 (1995).

${ }^{9}$ H. Kino and H. Fukuyama, J. Phys. Soc. Jpn. 65, 2158 (1996).

${ }^{10}$ M. Ogata, J. Phys. Soc. Jpn. 72, 1839 (2003).

${ }^{11}$ J. Y. Gan, Y. Chen, Z. B. Su, and F. C. Zhang, Phys. Rev. Lett. 94, 067005 (2005).

${ }^{12}$ T. Watanabe, H. Yokoyama, Y. Tanaka, J. I. Inoue, and M. Ogata, J. Phys. Soc. Jpn. 73, 3404 (2004).

${ }^{13}$ J. Y. Gan, F. C. Zhang, and Z. B. Su, Phys. Rev. B 71, 014508 (2005).
${ }^{14}$ B. J. Powell and R. H. McKenzie, Phys. Rev. Lett. 94, 047004 (2005).

${ }^{15}$ J. Liu, J. Schmalian, and N. Trivedi, Phys. Rev. Lett. 94, 127003 (2005).

${ }^{16}$ B. J. Powell and R. H. McKenzie, J. Phys.: Condens. Matter 18, R827 (2006).

${ }^{17}$ T. Watanabe, H. Yokoyama, Y. Tanaka, and J. I. Inoue, J. Phys. Soc. Jpn. 75, 074707 (2006).

${ }^{18}$ B. Kyung and A. M. S. Tremblay, Phys. Rev. Lett. 97, 046402 (2006).

${ }^{19}$ T. Watanabe, H. Yokoyama, Y. Tanaka, and J. Inoue, Phys. Rev. B 77, 214505 (2008).

${ }^{20}$ F. C. Zhang, Phys. Rev. Lett. 90, 207002 (2003).

${ }^{21}$ R. B. Laughlin, Philos. Mag. 86, 1165 (2006).

${ }^{22}$ B. A. Bernevig, R. B. Laughlin, and D. I. Santiago, Phys. Rev. Lett. 91, 147003 (2003).

${ }^{23}$ C. Gros, Ann. Phys. 189, 53 (1989).

${ }^{24}$ T. Komatsu, N. Matsukawa, T. Onoue, and G. Saito, J. Phys. Soc. Jpn. 65, 1340 (1996).

${ }^{25}$ C. N. Yang, Rev. Mod. Phys. 34, 694 (1962).

${ }^{26}$ C. Gros, Phys. Rev. B 38, 931 (1988). 